\title{
Retraction Note: NF-кB activation impairs somatic cell reprogramming in ageing
}

Clara Soria-Valles, Fernando G. Osorio, Ana Gutiérrez-Fernández, Alejandro De Los Angeles, Clara Bueno, Pablo Menéndez, José I. Martín-Subero, George Q. Daley, José M. P. Freije and Carlos López-Otín

Retraction of: Nature Cell Biology https://doi.org/10.1038/ncb3207, published online 27 July 2015.

We, the authors, are retracting this Article due to issues that have come to our attention regarding data availability, data description and figure assembly. Specifically, original numerical data are not available for the majority of the graphs presented in the paper. Although original data were available for most EMSA and immunoblot experiments, those corresponding to the published EMSA data of Supplementary Fig. 8a, the independent replicate immunoblots of Fig. 8b and Supplementary Fig. 1e, and the independent replicate EMSA data of Supplementary Figs $6 \mathrm{e}, 8 \mathrm{~b}, 8 \mathrm{c}$ and $8 \mathrm{~d}$, are unavailable. Mistakes were detected in the presentation of Figs $3 \mathrm{c}$, 4i and Supplementary Figs 6a, 8a, 8d, 9, and in some cases the $\beta$-actin immunoblots were erroneously described in the figure legends as loading controls, rather than as sample processing controls that were run on separate gels. Although we, the authors, believe that the key findings of the paper are still valid, given the issues with data availability we have concluded that the most appropriate course of action is to retract the Article. We deeply regret these errors and apologize to the scientific community for any confusion this publication may have caused. All authors agree with the retraction.

Published online: 17 December 2018

https://doi.org/10.1038/s41556-018-0259-0 